\title{
Testosterone treatment and risk of venous thromboembolism: population based case-control study
}

\author{
Carlos Martinez,, Samy Suissa, ${ }^{2}$ Stephan Rietbrock, ${ }^{1}$ Anja Katholing,, Ben Freedman, 3,4,5 \\ Alexander T Cohen, ${ }^{6}$ David J Handelsman³
}

${ }^{1}$ Institute for Epidemiology,

Statistics and Informatics

GmbH, 60388 Frankfurt,

Germany

${ }^{2}$ Centre For Clinical

Epidemiology, Lady Davis

Research Institute - Jewish

General Hospital, McGill

University, Montreal, Quebec

H3T 1E2, Canada

${ }^{3}$ ANZAC Research Institute,

University of Sydney, Concord

Hospital, Concord NSW 2139 ,

Australia

${ }^{4}$ Heart Research Institute,

Charles Perkins Centre,

University of Sydney, NSW 2006

Australia

${ }^{5}$ Concord Hospital Dept of

Cardiology, Sydney Medical

School, University of Sydney,

Sidney NSW 2006, Australia

${ }^{6}$ Department of Haematology,

Guy's and St Thomas' NHS

Foundation Trust, King's College

London, London, UK

Correspondence to: C Martinez carlos.martinez@pharmaepi.com

Additional material is published online only. To view please visit

the journal online.

Cite this as: BMJ 2016;355:i5968

http://dx.doi.org/10.1136/bmj.i5968

Accepted: 28 October 2016

\begin{abstract}
OBJECTIVE

To determine the risk of venous thromboembolism associated with use of testosterone treatment in men, focusing particularly on the timing of the risk.

DESIGN

Population based case-control study.

SETTING

370 general practices in UK primary care with linked hospital discharge diagnoses and in-hospital procedures and information on all cause mortality.

PARTICIPANTS

19215 patients with confirmed venous

thromboembolism (comprising deep venous

thrombosis and pulmonary embolism) and 909530

age matched controls from source population

including more than 2.22 million men between January 2001 and May 2013.
\end{abstract}

\section{EXPOSURE OF INTEREST}

Three mutually exclusive testosterone exposure groups were identified: current treatment, recent (but not current) treatment, and no treatment in the previous two years. Current treatment was subdivided into duration of more or less than six months.

\section{MAIN OUTCOME MEASURE}

Rate ratios of venous thromboembolism in association with current testosterone treatment compared with no treatment were estimated using conditional logistic regression and adjusted for comorbidities and all matching factors.

RESULTS

The adjusted rate ratio of venous thromboembolism was 1.25 (95\% confidence interval 0.94 to 1.66 ) for current versus no testosterone treatment. In the first six months of testosterone treatment, the rate ratio of venous thromboembolism was 1.63 (1.12 to 2.37), corresponding to 10.0 (1.9 to 21.6$)$ additional venous

\section{WHAT IS ALREADY KNOWN ON THIS TOPIC}

An increase in prescribing of testosterone in men has occurred over the first decade of this century, mainly for sexual dysfunction and/or decreased energy

Studies have reported contradictory results on an association between

testosterone use and the risk of venous thromboembolism

The effect of timing and duration of testosterone use on the risk of venous

thromboembolism was not studied and may explain these contradictory reports

\section{WHAT THIS STUDY ADDS}

This observational study suggests an increased risk of venous thromboembolism soon after the start of testosterone use, which peaks in the first six months

thromboembolisms above the base rate of 15.8 per 10000 person years. The rate ratio after more than six months' treatment was 1.00 (0.68 to 1.47$)$, and after treatment cessation it was 0.68 ( 0.43 to 1.07 ). Increased rate ratios within the first six months of treatment were observed in all strata: the rate ratio was 1.52 (0.94 to 2.46) for patients with pathological hypogonadism and 1.88 (1.02 to 3.45) for those without it, and 1.41 ( 0.82 to 2.41 ) for those with a known risk factor for venous thromboembolism and 1.91 (1.13 to 3.23) for those without one.

\section{CONCLUSIONS}

Starting testosterone treatment was associated with an increased risk of venous thromboembolism, which peaked within six months and declined thereafter.

\section{Introduction}

A striking increase in prescribing of testosterone occurred over the first decade of this century, with a 10 -fold increase in testosterone prescriptions per capita in the United States (excluding compounding pharmacies). ${ }^{1}$ The 40 -fold increase in Canada, which includes internet pharmacies providing additional testosterone sales, suggests that this may be an underestimate. Yet pathological hypogonadism, defined as recognised pathological disorders of the male reproductive system, remains the sole unequivocal indication for testosterone treatment. ${ }^{2}$ Hence this upsurge in medical testosterone use over the first decade of the century without new proven indications is for unclear and questionable indications, probably sexual dysfunction and/or decreased energy/vitality. ${ }^{34}$ Since then, a recent study has shown only a modest and poorly sustained increase in sexual activity, but not in vitality or physical activity, in men without pathological hypogonadism over the age of 65 years treated with testosterone. ${ }^{5}$

An important consequence of unjustified testosterone treatment in men with an increased underlying cardiovascular risk is the possibility of an excess of cardiovascular events with testosterone treatment. One study reported an excess of cardiovascular events in older men without pathological hypogonadism, ${ }^{6}$ but subsequent meta-analyses of randomised placebo controlled trials of adverse cardiovascular effects from testosterone treatment have provided discrepant interpretations. ${ }^{7-12}$ However, the overall risk ratios were 1.0 or greater in each trial, suggesting that these relatively short term analyses with wide confidence intervals were still too under-powered to be decisive. ${ }^{13}$ On the basis of congruent considerations, in June 2014 the US Food and Drug Administration and Health Canada required a new general label warning in the product information of all approved testosterone products 
about risk of venous thromboembolism after testosterone treatment. ${ }^{1415}$ Since then, one small meta-analysis of randomised controlled trials of testosterone found it to be associated with a higher risk of venous thromboembolism. ${ }^{16}$ A cohort study and a case-control study did not show evidence of an association between testosterone use and an increased risk of venous thromboembolism. ${ }^{1718}$ None of the previous studies, however, investigated the timing and duration of testosterone use, which could have masked a risk of venous thromboembolism soon after the start of treatment, as seen with oral contraceptives. ${ }^{19}$

In the absence of large randomised trials, we used a large UK database to investigate the association between testosterone use and the risk of venous thromboembolism in men, focusing particularly on the timing of exposure to testosterone and the presence of risk factors for venous thromboembolism.

\section{Methods}

\section{Study setting and design}

The source population consisted of all patients in the UK Clinical Practice Research Datalink (CPRD) eligible for additional data from the Hospital Episodes Statistics and the Office for National Statistics. The CPRD is based on patients' encounters with the general practices. Data are recorded electronically and include patients' demographics; lifestyle information; medical history; symptoms, signs, and diagnoses recorded with Read medical codes; laboratory tests; immunisations; unstructured medical notes; letters to and from secondary care; and prescriptions issued by the general practitioner. The CPRD is broadly representative of the UK population in terms of age, sex, and ethnicity. More than $98 \%$ of the UK population are registered with primary care general practitioners, who act as gatekeepers to the health system. The CPRD undertakes assessment of data quality at the patient level and at the practice level to assign patients and the latest date at which practices meet minimum quality criteria. ${ }^{20}$ It has been validated in venous thromboembolism research. ${ }^{2122}$ Hospital Episodes Statistics include dates of hospital admission and discharge, primary and other main reasons for treatment recorded with ICD-10 (international classification of diseases, 10th revision), and surgical operations and procedures performed during the hospital stay. Office for National Statistics data contain the date and cause of death as recorded with ICD-10 in death certificates.

We did a population based case-control study of all men aged 20 to 89 years in CPRD general practices satisfying the quality standard for research between 1 January 2001 and 31 May 2013.

\section{Case definition}

We identified all cases with a diagnosis of a venous thromboembolism between January 2001 and May 2013 from the study population. We applied a validated algorithm for venous thromboembolism to identify the first occurrence. ${ }^{22}$ Venous thromboembolism comprised pulmonary embolism and deep vein thrombosis. Deep vein thrombosis included thrombosis of the deep veins of lower and upper limbs and thrombosis of the pelvic veins and vena cava. We developed an algorithm for venous thromboembolism based on events identified from causes of death, hospital discharge diagnoses, general practitioner based medical records, anonymised clinical notes, and search of general practitioner recorded medical notes for word strings indicative of venous thromboembolism and use of oral and parenteral anticoagulants. We also used anonymised clinical notes, letters, and discharge summaries to validate the diagnosis. Venous thromboembolisms diagnosed by general practitioners required use of oral anticoagulants or at least three international normalised ratio tests within seven days before to 60 days after the initial event or at least two prescriptions for low molecular weight heparin within seven to 60 days after the venous thromboembolism. We prioritised diagnoses of venous thromboembolism in one data source over those in another data source if the first event was recorded in more than one data source at the same time. In those cases, we prioritised venous thromboembolisms according to the following hierarchy: manual review of clinical notes, Office for National Statistics, Hospital Episodes Statistics, general practitioner, and word strings for venous thromboembolism. We assessed the validity of the venous thromboembolism algorithm by reviewing anonymised clinical and hospital discharge letters from the entire CPRD for those with a recording of venous thromboembolism.

The sensitivity for detecting venous thromboembolism was $92.6 \%$, and the specificity was $98.6 \% .^{22}$ The first occurrence of VTE during the study period defined the case, and we designated the date of this event as the index date. All cases had at least two years of up-to-standard history in the CPRD before the index date and no history of a previous venous thromboembolism. We classified all venous thromboembolism events in the study cohort according to the presence or absence of each of the following risk factors: cancer, surgery, trauma, or a medical condition in the 90 days before the index event and history of cancer recorded more than 90 days before the index day. ${ }^{23}$ Medical illness refers to acutely ill medical patients and comprised a primary hospital discharge diagnosis for any of the following conditions recorded in the 90 days before the index venous thromboembolism: acute stroke, heart failure, rheumatological conditions, inflammatory bowel disease, cellulitis of skin, gut infections, lower and upper urinary tract infections, osteoporosis, acute respiratory failure, asthma, bronchiectasis, bronchiolitis, bronchitis, chronic obstructive pulmonary disease, influenza, and pneumonia. ${ }^{24}$ Cancer was defined as an admission to hospital with a primary diagnosis of cancer (excluding non-melanoma skin cancer), radiation treatment, chemotherapy, or bone marrow transplantation.

\section{Selection of controls}

Because exposure to testosterone is rare, for each venous thromboembolism case we randomly selected up to 50 matched controls among people in the source population on the case's index date, which became the 
index date for those matched controls. For each case, controls were matched on year of birth, all known risk factors for venous thromboembolism in the 90 days before the index day (that is, surgical procedures, medical illness, trauma, active cancer), history of cancer 91 or more days before the index date, and history of pathological hypogonadism. This last consisted of primary or secondary hypogonadism on the basis of the presence of testicular or hypothalamic-pituitary pathologies, respectively, recorded any time before the index date. The matching factors year of birth, history of primary or secondary hypogonadism, and known risk factors for venous thromboembolism in the 90 days before the index day are known or suspected confounding factors that are or may be causes of prescriptions for testosterone or of venous thromboembolism. Venous thromboembolism cases were eligible as potential controls until they became cases, and all matches had to be at risk of an incident venous thromboembolism. Controls also had to have two years of up-to-standard history in the CPRD and no history of a venous thromboembolism before the index date.

\section{Exposures}

For all cases and their matched controls, we estimated exposure from all prescriptions for a testosterone product issued before the index date. We considered a man to be exposed for the length of a prescription plus a 30 day grace period. We considered men to be currently exposed if they had a prescription whereby the use (prescription length+30 days) extended to or beyond the index date. Current treatment was stratified into the time since start of testosterone treatment up to six months or more than six months before the index day. Recent use was use that (including the 30 day grace period) ended at a point between two years before and one day before the index date. Not exposed was the reference category for all comparisons and comprised all patients with use that ended more than two years before the index date plus those who never received testosterone. We calculated the start and end of testosterone treatment for each case and control by using the prescribed product to account for its dose (mg), formulation (capsules, injectable, transdermal gel or patch, subcutaneous implants) and route of administration (oral, intramuscular, transdermal). We calculated product specific duration of exposure to testosterone by its prescribed quantity and daily supply. We defined the date of a repeat prescription as the date of the next prescription in the respective sequence, which must have occurred within the coverage days of the first prescription, defined by the calculated duration of exposure plus a 30 day grace period. The grace period was to account for residual effects of testosterone or imperfect adherence to the prescribed testosterone regimen (for example, late make-up for missed doses and/or remaining drug after apparent date of cessation).

The coverage days for all testosterone products were based on expert opinion, and the median time between product specific prescriptions was 14 or 84 days for shorter and longer acting testosterone injections, 150 days for implants, and 30 days for products with another (oral, transdermal) route of administration.

In a sensitivity analysis, we repeated the main analysis using a 15 and 60 day grace period. No testosterone treatment (within the previous two years) was the reference category for all comparisons.

\section{Covariates}

Covariates included all matching factors used in the design as well as the following additional covariates: polycythaemia, chronic pulmonary disease, diabetes, congestive heart failure, myocardial infarction, peripheral vascular disease, stroke, and history of prothrombotic disease. For all cases and controls without pathological hypogonadism, the following comorbidities, which sometimes lead to empirical testosterone treatment, were assessed if recorded before the index date: sexual dysfunction, osteoporosis, and infertility; and the following symptoms and complaints were assessed if recorded in the year before the index date: hot flushes, loss of appetite, and a group of symptoms comprising tiredness, lethargy, and depression. Other covariates were body mass index (using cut-offs of 18.5, 25.0, 30.0, and 35.0), smoking and drinking status based on the last information before or on the index date, socioeconomic status, and prescriptions for the following drugs within 90 days of the index date: megestrol, non-steroidal anti-inflammatory drugs, systemic corticosteroids, and antiplatelets.

\section{Statistical analysis}

We used conditional logistic regression to calculate crude and adjusted odds ratios that are estimates of the incidence rate ratios. The main analysis included current and recent testosterone treatment as of the index date compared with no testosterone treatment. In addition to the matching factors, all rate ratios were adjusted for residual imbalance of all specified covariates among cases and controls. We did stratified analyses for patients with a known risk factor within 90 days before the index day or with a history of cancer or pathological hypogonadism, and for route of administration. To obtain an absolute measure of the excess incidence rate, we transformed the adjusted rate ratio to a rate difference by using the proportion of controls exposed to testosterone, the adjusted rate ratio associated with testosterone use, and the incidence rate of venous thromboembolism in our study population. ${ }^{1925}$ We used quadratic splines to illustrate the rate ratio of venous thromboembolism as a function of duration of testosterone treatment among men currently treated with testosterone.

To assess the robustness of our results, we did sensitivity analyses exploring the effect of potential misclassification of current testosterone exposure on the estimated rate ratios by assigning grace periods of 15 and 60 days rather than 30 days or by using the entire patient's history in the CPRD for the definition of no testosterone treatment or for the stratification of current testosterone therapy according to the start of treatment using three or nine months rather than six months. We used Stata MP version 13.1 for all statistical procedures. 


\section{Patient involvement}

No patients were involved in setting the research question or the outcome measures, nor were they involved in developing plans for design or implementation of the study. No patients were asked to advise on interpretation or writing up of results. There are no plans to disseminate the results of the research to study participants or the relevant patient community.

\section{Results}

The source population consisted of 2.92 million men. During a total of 12.1 million person years of follow-up, 19246 men had an incident confirmed diagnosis of venous thromboembolism (8394 deep venous thromboses, 10787 pulmonary embolisms, and 65 unspecified venous thromboembolisms according to the case finding algorithm detailed in fig 1), for an incidence rate of venous thromboembolism of 15.8 (95\% confidence interval 15.6 to 16.0 ) per 10000 person years.

A total of 909530 controls were matched to 19215 venous thromboembolism cases on age and all predefined independent risk factors for venous thromboembolism. No matches were found for 31 cases. Table 1 confirms that the venous thromboembolism cases and their matched controls were identical in age and the predefined matching. Cases were more often obese (body mass index $\geq 30: 27.0 \% v 20.6 \%$ ) and had more comorbidities, notably polycythaemia, chronic pulmonary disease, congestive heart failure, myocardial infarction, and peripheral vascular disease than did the controls. Additionally, corticosteroids, non-steroidal anti-inflammatory drugs, and antiplatelets were used more frequently among cases than controls (table 1).

On the index date, $1251(0.14 \%)$ of the controls were current testosterone users and $846(0.09 \%)$ were recent users. Of the 19215 cases, 69 venous thromboembolisms $(0.36 \%$; 28 deep vein thromboses and 41 pulmonary embolisms) occurred during current testosterone treatment and 22 (0.11\%; 7 deep vein thromboses and 15 pulmonary embolisms) after recent treatment (table 2). Of the 1251 controls currently treated with testosterone, $678(54.2 \%)$ used intramuscular preparations,

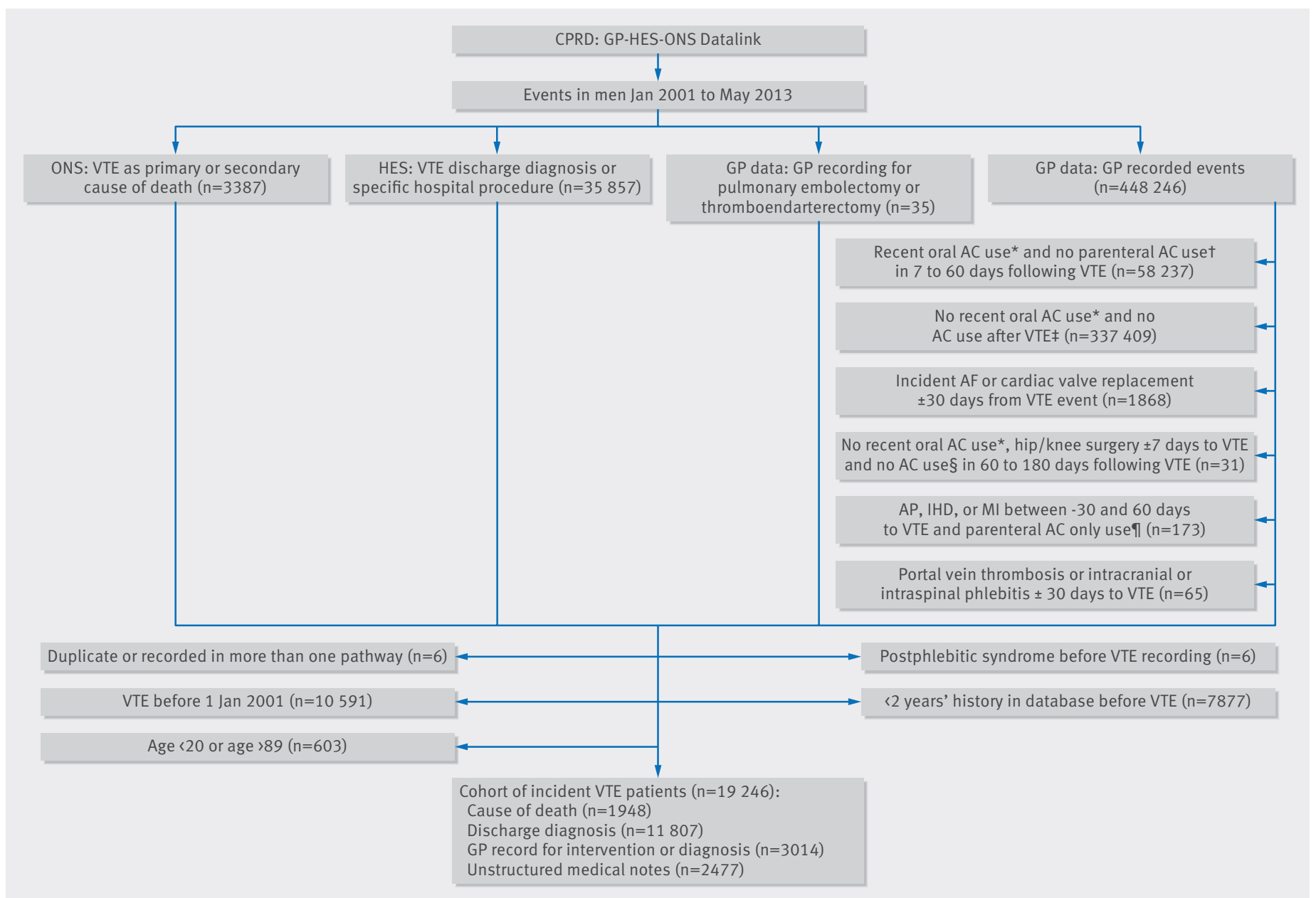

Fig 1 Ascertainment of first venous thromboembolism (VTE) among 2.92 million men in CPRD-HES-ONS January 2001 to May 2013 . AC=anticoagulant; $\mathrm{AF}=$ atrial fibrillation; $\mathrm{AP}=$ angina pectoris; $\mathrm{CRPD}=$ Clinical Practice Research Datalink; $\mathrm{GP}=$ general practitioner; HES=Hospital Episode Statistics; $\mathrm{IHD}=$ =ischaemic heart disease; INR=international normalised ratio; $\mathrm{LMWH}=$ low molecular weight heparin; MI=myocardial infarction; ONS=0ffice for National Statistics. *Prescription for oral AC or $\geq 3$ INR tests 31-180 days before VTE. +LMWH prescription. $¥ 0$ ral AC use or $\geq 3$ INR tests within -7 to 60 days of VTE or $\geq 2$ LMWH prescriptions within 7 to 60 days after VTE. §Oral AC prescription, LMWH prescription, or INR test. ๆLMWH prescription and no oral AC prescription within -30 to 60 days of VTE 
Table 1 | Characteristics of 19244 case patients with venous thromboembolism (VTE) and 928299 control patients from Clinical Practice Research Datalink. Values are numbers (percentages) unless stated otherwise

\begin{tabular}{|c|c|c|}
\hline & $\begin{array}{l}\text { VTE cases } \\
(\mathrm{n}=19215)\end{array}$ & $\begin{array}{l}\text { Matched controls } \\
(\mathrm{n}=909530)\end{array}$ \\
\hline \multicolumn{3}{|l|}{ Age* $^{*}$} \\
\hline Mean (SD) age, years & $64.8(15.2)$ & $64.8(15.2)$ \\
\hline \multicolumn{3}{|l|}{ Age group, years: } \\
\hline $18-29$ & $460(2.4)$ & $21279(2.4)$ \\
\hline 30-39 & $1058(5.5)$ & $48227(5.5)$ \\
\hline $40-49$ & $1782(9.3)$ & $80120(9.3)$ \\
\hline $50-59$ & $2872(14.9)$ & 132735 (14.9) \\
\hline $60-69$ & $4539(23.6)$ & $219339(23.6)$ \\
\hline $70-79$ & $5166(26.9)$ & $250148(26.9)$ \\
\hline$\geq 80$ & $3338(17.4)$ & $157682(17.4)$ \\
\hline \multicolumn{3}{|l|}{ Body mass index } \\
\hline Known & $16338(85.0)$ & $767402(84.4)$ \\
\hline Mean (SD) & $27.7(5.4)$ & $26.8(4.6)$ \\
\hline Median (interquartile range) & $27(24-30)$ & $26(24-29)$ \\
\hline \multicolumn{3}{|l|}{ BMI group: } \\
\hline$<18.5$ & $282(1.7)$ & $11995(1.6)$ \\
\hline 18.5 to $<25.0$ & $4824(29.5)$ & $266707(34.8)$ \\
\hline 25.0 to $<30.0$ & $6823(41.8)$ & $330614(43.0)$ \\
\hline 30 to $<35.0$ & $2986(18.3)$ & $120151(15.6)$ \\
\hline$\geq 35$ & $1423(8.7)$ & $37935(5.0)$ \\
\hline Unknown & $2877(15.0)$ & $142128(15.6)$ \\
\hline \multicolumn{3}{|l|}{ Alcohol } \\
\hline Known & $16424(85.5)$ & $771548(84.8)$ \\
\hline Never drinker & $2042(12.4)$ & $91655(12.0)$ \\
\hline Ex-drinker & $602(3.7)$ & $23770(3.1)$ \\
\hline Current drinker & $13780(83.9)$ & $656123(84.9)$ \\
\hline \multicolumn{3}{|l|}{ Smoking } \\
\hline Known & $18271(95.1)$ & 852158 (93.7) \\
\hline Never smoker & $7390(40.4)$ & $362319(42.4)$ \\
\hline Ex-smoker & 7106 (38.9) & $323555(37.8)$ \\
\hline Current smoker & $3775(20.7)$ & $166284(19.8)$ \\
\hline \multicolumn{3}{|l|}{ Socioeconomic status } \\
\hline Known SES & $19133(99.6)$ & $906712(99.7)$ \\
\hline \multicolumn{3}{|l|}{ Fifth of SES: } \\
\hline First (least deprived) & $4253(22.2)$ & $214187(23.4)$ \\
\hline Second & $4546(23.8)$ & $223713(24.6)$ \\
\hline Third & $3828(20.0)$ & $185904(20.5)$ \\
\hline Fourth & $3582(18.7)$ & $160693(17.8)$ \\
\hline Fifth (most deprived) & $2924(15.3)$ & $122215(13.7)$ \\
\hline Unknown SES & $82(0.4)$ & $2818(0.3)$ \\
\hline \multicolumn{3}{|l|}{ Known VTE risk factors* } \\
\hline Any known VTE risk factor & $9445(49.1)$ & $421069(49.1)$ \\
\hline \multicolumn{3}{|l|}{ Individual risk factors: } \\
\hline Surgery (90 days) & $3675(19.1)$ & 178441 (19.1) \\
\hline Medical illness (90 days) & $1398(7.3)$ & $55253(7.3)$ \\
\hline Trauma (90 days) & $161(0.8)$ & $1309(0.8)$ \\
\hline Cancer history (>90 days) & $672(3.5)$ & $32063(3.5)$ \\
\hline Active cancer (90 days) & 3539 (18.4) & $154003(18.4)$ \\
\hline No known risk factor & $9770(50.8)$ & $488461(50.8)$ \\
\hline \multicolumn{3}{|l|}{ Comorbidities } \\
\hline Polycythaemia & $165(0.9)$ & $3305(0.4)$ \\
\hline Chronic pulmonary disease & 4969 (25.9) & $185346(20.7)$ \\
\hline Diabetes & $2818(14.7)$ & $125749(13.9)$ \\
\hline Congestive heart failure & $2195(11.4)$ & $58391(6.6)$ \\
\hline Myocardial infarction & $2027(10.5)$ & $78850(8.7)$ \\
\hline Peripheral vascular disease & $1889(9.8)$ & $63428(7.1)$ \\
\hline Stroke & $605(3.1)$ & $24203(2.8)$ \\
\hline Pathological hypogonadism* & 740 (3.9) & $19403(3.9)$ \\
\hline Primary hypogonadism & $635(3.3)$ & $18325(3.6)$ \\
\hline Secondary hypogonadism & $105(0.5)$ & $1078(0.3)$ \\
\hline
\end{tabular}

(Continued)
$453(36.2 \%)$ transdermal preparations, $23(1.8 \%)$ implants, and 97 (7.8\%) oral preparations (table 3). The median duration of the last prescription before the index date was 85 days for intramuscular administrations, 31 days for patches, gel, or oral testosterone products, and 151 days for subcutaneous testosterone implants.

Overall crude rate ratios were greater than adjusted rate ratios, with secondary hypogonadism, corticosteroids, and body mass index contributing most to the adjustment. The adjusted rate ratio of venous thromboembolism associated with current testosterone treatment was 1.25 (95\% confidence interval 0.94 to 1.66 ) relative to no testosterone treatment (table 2), corresponding to an adjusted excess incidence rate of 3.9 ( -0.9 to 10.4 ) additional venous thromboembolisms per 10000 person years of current testosterone use. The rate ratio of venous thromboembolism associated with starting a new course of testosterone treatment within six months was 1.63 (1.12 to 2.37), corresponding to an excess incidence rate of 10.0 (1.9 to 21.6) additional venous thromboembolisms per 10000 person years, whereas the rate ratio for treatment started longer than six months before the index date was 1.00 (0.68 to 1.47). The rate ratio for recent use was 0.68 (0.43 to 1.07) (table 2). Figure 2 shows the quadratic spline curve and $95 \%$ confidence limits of the rate ratio as a function of time since starting the current testosterone treatment, showing that the risk of venous thromboembolism peaks at about three months after starting testosterone treatment and declines progressively thereafter towards the pre-treatment baseline.

Stratification according to the presence of pathological hypogonadism yielded an adjusted rate ratio of venous thromboembolism of 1.69 (1.09 to 2.63) for current testosterone users without pathological hypogonadism and 1.88 (1.02 to 3.45) for those who started a new course of testosterone treatment within six months (table 2). In contrast, the rate ratio among patients with pathological hypogonadism was not significant for current testosterone treatment 1.08 ( 0.75 to 1.55 ), and it was 1.52 (0.94 to 2.46) for treatment started less than six months before (table 2). The increased rate ratio among patients without pathological hypogonadism and with current testosterone use corresponded to an adjusted rate difference of 10.9 (1.4 to 25.7) additional venous thromboembolisms per 10000 person years of current testosterone use.

Among patients with at least one known risk factor for venous thromboembolism, the rate ratio was 0.99 (0.65 to 1.49) for current testosterone users and 1.41 (0.82 to 2.41) for those who had started a new course of testosterone treatment within the previous six months. Among those without known risk factors, the rate ratio was higher at 1.57 (1.06 to 2.32) for current testosterone users and 1.91 (1.13 to 3.23) for those who had started a new course of testosterone treatment within the previous six months (table 3 ; fig 2). Stratification by route of administration yielded similar rate ratios for current intramuscular, transdermal, and oral testosterone use, with estimates ranging from 1.2 to 1.4 for patients currently treated with testosterone (table 3 ). 
Table 1 | Characteristics of 19244 case patients with venous thromboembolism (VTE) and 928299 control patients from Clinical Practice Research Datalink. Values are numbers (percentages) unless stated otherwise

\begin{tabular}{|c|c|c|}
\hline & $\begin{array}{l}\text { VTE cases } \\
(\mathrm{n}=19215)\end{array}$ & $\begin{array}{l}\text { Matched controls } \\
(\mathrm{n}=909530)\end{array}$ \\
\hline No pathological hypogonadism & $18475(96.1)$ & $890127(96.2)$ \\
\hline Sexual dysfunctionf & $2734(14.2)$ & $131767(14.1)$ \\
\hline Tiredness/lethargy/depression§ & $4136(21.5)$ & $174136(18.8)$ \\
\hline Others: & $835(4.3)$ & $32338(3.6)$ \\
\hline Osteoporosis $¥$ & $449(2.3)$ & $19411(2.2)$ \\
\hline Infertility & $111(0.6)$ & $6044(0.7)$ \\
\hline Loss of appetite§ & $206(1.1)$ & $4926(0.6)$ \\
\hline Hot flushes§ & $83(0.4)$ & $2476(0.3)$ \\
\hline \multicolumn{3}{|l|}{ Concomitant drugs } \\
\hline Corticosteroids & $2241(11.7)$ & $48053(5.6)$ \\
\hline Megestrol & $20(0.1)$ & $400(0.0)$ \\
\hline NSAIDs & $2721(14.2)$ & $81715(9.1)$ \\
\hline Antiplatelets & $4617(24.0)$ & $207544(22.7)$ \\
\hline
\end{tabular}

To account for varying number of controls matched to each case, all means, standard deviations, and percentages for controls were weighted by inverse of number of controls in each matched case-control set. $\mathrm{BMI}=$ body mass index; NSAID=non-steroidal anti-inflammatory drug; SES=socioeconomic status.

*Matching factor.

tKnown VTE risk factors defined as hospital diagnosis for medical condition or trauma or in-hospital surgical procedure in 90 days before index VTE or history of cancer recorded any time before index date.

$\ddagger$ Recorded any time before index date.

$\S$ Recorded in year before index date.

IRecorded within 90 days before index date.

Sensitivity analyses using three, six, and nine month cut-off periods for start of testosterone treatment among current users and applying a 15, 30, and 60 day grace period to define continuous exposure, compared with the six month cut-off and 30 day grace period used in the main analysis, showed similar findings (supplementary table). We did another analysis by reallocating patients with use of testosterone two or more years before the index day from the unexposed group to the "recent testosterone" group. This sensitivity analysis did not alter the findings.

\section{Discussion}

This study provides the first evidence for a differential in an adverse effect of testosterone treatment in men with and without pathological hypogonadism, which peaks in the first six months and declines thereafter. The 63\% increase in risk in the first six months of testosterone use corresponds to 10.0 (1.9 to 21.6) additional venous thromboembolisms above the base rate of 15.8 per 10000 person years. Although at variance with another recent report, ${ }^{18}$ these findings support the addition of the general warning for risk of venous thromboembolism with testosterone products required recently by the US Food and Drug Administration, although they highlight the fact that that this increased risk is transient.

\section{Strengths and weaknesses of study}

The strengths of this study include the use of a validated venous thromboembolism algorithm, a non-venous thromboembolism cohort matched on known and suspected confounding factors that are or may be causes of prescriptions for testosterone or of venous thromboembolism, and the availability of full prescription data allowing investigation of risk of venous thromboembolism by time since starting or stopping testosterone treatment. ${ }^{22}$ In addition, we were able to adjust for other potential confounders known to be associated with the risk of future venous thromboembolism and to reduce confounding by indication by matching on history of hypogonadism. Furthermore, we verified the findings by a variety of sensitivity analyses showing similar results.

Table 2 | Crude and adjusted rate ratios of venous thromboembolism (VTE) stratified by history of pathological hypogonadism

\begin{tabular}{|c|c|c|c|c|}
\hline & \multicolumn{2}{|l|}{ No (\%) } & \multicolumn{2}{|l|}{ Rate ratio $(95 \% \mathrm{Cl})$} \\
\hline & Cases & Controls & Crude & Adjusted* \\
\hline Complete study cohort & $(n=19215)$ & $(n=909530)$ & & \\
\hline No testosterone treatment & $19124(99.53)$ & $907433(99.77)$ & 1 & 1 \\
\hline Current testosterone treatment† & $69(0.36)$ & $1251(0.14)$ & 1.84 (1.42 to 2.38$)$ & 1.25 (0.94 to 1.66$)$ \\
\hline Started $\leq 6$ months before & $36(0.19)$ & $529(0.06)$ & 2.26 (1.58 to 3.23$)$ & $1.63(1.12$ to 2.37$)$ \\
\hline Started $>6$ months before & $33(0.17)$ & $722(0.08)$ & $1.53(1.06$ to 2.21$)$ & $1.00(0.68$ to 1.47$)$ \\
\hline Recent testosterone use $\ddagger$ & $22(0.11)$ & $846(0.09)$ & 0.88 (0.57 to 1.36$)$ & $0.68(0.43$ to 1.07$)$ \\
\hline Without pathological hypogonadism & $(n=18475)$ & $(n=890127)$ & & \\
\hline No testosterone treatment & $18447(99.85)$ & $888997(99.87)$ & 1 & 1 \\
\hline Current testosterone treatment $\dagger$ & $21(0.11)$ & $530(0.06)$ & $1.91(1.23$ to 2.96$)$ & 1.69 (1.09 to 2.63$)$ \\
\hline Started $\leq 6$ months before & $11(0.06)$ & $252(0.03)$ & $2.06(1.12$ to 3.77$)$ & $1.88(1.02$ to 3.45$)$ \\
\hline Started $>6$ months before & $10(0.05)$ & $278(0.03)$ & 1.77 (0.94 to 3.33$)$ & 1.53 (0.81 to 2.88$)$ \\
\hline Recent testosterone use $\neq$ & $7(0.04)$ & $600(0.07)$ & 0.54 (0.26 to 1.15$)$ & $0.50(0.24$ to 1.05$)$ \\
\hline With pathological (primary or secondary) hypogonadism & $(n=740)$ & $(n=19403)$ & & \\
\hline No testosterone treatment & $677(91.49)$ & $18436(95.02)$ & 1 & 1 \\
\hline Current testosterone treatment ${ }^{\dagger}$ & $48(6.49)$ & $721(3.72)$ & 1.81 (1.31 to 2.50$)$ & $1.08(0.75$ to 1.55$)$ \\
\hline Started $\leq 6$ months before & $25(3.38)$ & $277(1.43)$ & 2.39 (1.53 to 3.75$)$ & 1.52 (0.94 to 2.46$)$ \\
\hline Started $>6$ months before & $23(3.11)$ & $444(2.29)$ & 1.44 (0.92 to 2.26$)$ & $0.82(0.50$ to 1.32$)$ \\
\hline Recent testosterone use $\ddagger$ & $15(2.03)$ & $246(1.27)$ & 1.30 (0.74 to 2.28$)$ & 0.84 (0.46 to 1.52$)$ \\
\hline
\end{tabular}

*Adjusted for age, history of primary or secondary hypogonadism, surgical procedures, medical illness, trauma, and active cancer in 90 days before index date and for history of cancer $\geq 91$ days before index date (matching factors); for smoking, body mass index, alcohol, socioeconomic status, any history of polycythaemia, chronic pulmonary disease, diabetes, congestive heart

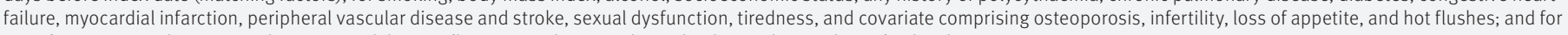
use of corticosteroids, megestrol, non-steroidal anti-inflammatory drugs, and antiplatelets within 90 days of index date.

tDefined as prescription for which duration included index date.

¥Defined as use that ended between two years and one day before index date. 


\begin{tabular}{|c|c|c|c|c|}
\hline & \multicolumn{2}{|l|}{ No (\%) } & \multicolumn{2}{|l|}{ Rate ratio $(95 \% \mathrm{Cl})$} \\
\hline Route of administration & $(n=19215)$ & $(n=909530)$ & & \\
\hline No recent testosterone treatment & $19124(99.53)$ & $907433(99.77)$ & 1 & 1 \\
\hline Transdermal & $24(0.12)$ & $453(0.05)$ & 1.87 (1.21 to 2.87$)$ & 1.31 (0.84 to 2.04) \\
\hline Oral & $5(0.03)$ & $97(0.01)$ & 1.76 (0.69 to 4.53) & $1.32(0.49$ to 3.55$)$ \\
\hline Implant & 0 & 23 & - & - \\
\hline Without known VTE risk factors & $(n=9770)$ & $(n=488461)$ & & \\
\hline No testosterone treatment & $9726(99.55)$ & $487270(99.76)$ & 1 & 1 \\
\hline Recent testosterone use§ & $7(0.07)$ & $407(0.08)$ & $0.88(0.42$ to 1.86$)$ & 0.62 (0.29 to 1.33$)$ \\
\hline With known VTE risk factors & $(n=9445)$ & $(n=421069)$ & & \\
\hline No testosterone treatment & $9398(99.50)$ & $420163(99.78)$ & 1 & 1 \\
\hline Current testosterone treatment $\dagger$ & $32(0.34)$ & $467(0.11)$ & $1.36(0.92$ to 2.00$)$ & 0.99 (0.65 to 1.49) \\
\hline Started $\leq 6$ months before & $19(0.20)$ & $208(0.05)$ & 1.89 (1.13 to 3.15) & 1.41 (0.82 to 2.41) \\
\hline Started $>6$ months before & $13(0.14)$ & $259(0.06)$ & $0.96(0.53$ to 1.73$)$ & 0.68 (0.37 to 1.27$)$ \\
\hline Recent testosterone use $\S$ & $15(0.16)$ & $439(0.10)$ & 0.87 (0.51 to 1.49) & $0.70(0.39$ to 1.23$)$ \\
\hline \multicolumn{5}{|c|}{$\begin{array}{l}\text { *Adjusted for age, history of primary or secondary hypogonadism, surgical procedures, medical illness, trauma, and active cancer in } 90 \text { days before } \\
\text { index date and for history of cancer } \geq 91 \text { days before index date (matching factors); for smoking, body mass index, alcohol, socioeconomic status, any } \\
\text { history of polycythaemia, chronic pulmonary disease, diabetes, congestive heart failure, myocardial infarction, peripheral vascular disease and stroke, } \\
\text { sexual dysfunction, tiredness, and covariate comprising osteoporosis, infertility, loss of appetite, and hot flushes; and for use of corticosteroids, } \\
\text { megestrol, non-steroidal anti-inflammatory drugs, and antiplatelets within } 90 \text { days of index date. } \\
\text { tDefined as prescription for which duration included index date. } \\
\text { ‡Defined as hospital diagnosis of medical condition or trauma or in-hospital surgical procedure in } 90 \text { days before index VTE, or history of cancer } \\
\text { recorded any time before index date. } \\
\S \text { Defined as use that ended between two years and one day before index date. }\end{array}$} \\
\hline
\end{tabular}

The observed difference in risk of venous thromboembolism in current users who started testosterone use more than six months before the index date between those with and those without pathological hypogonadism may be a genuine finding or may be due to chance alone, but two other possible explanations exist. The first is depletion of susceptible patients, ${ }^{26}$ with a different proportion of very long term testosterone users among those with and those without pathological hypogonadism (testosterone treatment is lifelong for pathological hypogonadism, ${ }^{2}$ whereas for men without pathological hypogonadism it is characteristically short term $)^{27}$. The second is effect measure modification due to different baseline risks of venous thromboembolism among patients with and without pathological hypogonadism, ${ }^{28}$ which could affect the rate ratio, although no independent evidence exists for a higher underlying risk in men with pathological hypogonadism.

The limitations of this study include those seen in all observational studies: unmeasured or residual confounding or hidden bias that might exist undetected. To minimise residual confounding, we also matched cases to controls on history of individual known risk factors for venous thromboembolism including a medical illness, surgery and trauma, and active cancer within 90 days of the index day. Stratification by presence and absence of known risk factors for venous thromboembolism showed non-significant differences between patients with and without the known risk factor. Although the rate ratios were not statistically different, the difference in magnitudes of effect may be clinically relevant (1.91 $v 1.41)$.
Case-control studies are prone to time window bias if the cases and controls are not defined by the same risk sets and the time windows for measuring exposure in cases and controls are not equal. In this study, we avoided time window bias by handling cases and controls in exactly the same way.

To mitigate the effect of polycythaemia as a potential mediator as well as of prostate cancer, a contraindication for testosterone use that could have altered the testosterone exposure status, we did sensitivity analysis without additional adjustment for polycythaemia and excluding all cases and controls with a history of prostate cancer. The study results remained basically unchanged. Nevertheless, unmeasured confounding remains a limitation. Another limitation is the potential

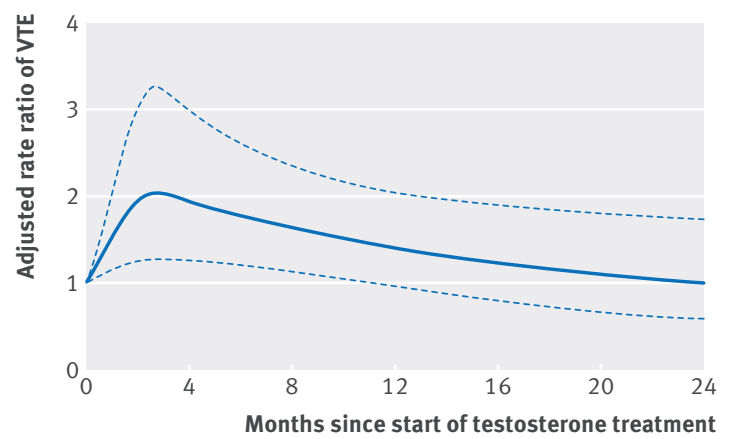

Fig 2 | Adjusted rate ratio of venous thromboembolism (VTE) and $95 \%$ confidence limits by time on current testosterone treatment. Testosterone treatment includes first time and repeat testosterone use 
for misclassification of exposure due to residual effects of the drug, remaining drug, and/or lack of adherence to treatment after apparent cessation. For this reason, we included a 30 day grace period in the original analysis, and a further sensitivity analysis using a shorter (15 day) and extended (60 day) grace period did not alter the findings.

The sensitivity of the algorithm for detecting venous thromboembolism was $92.6 \%$, which implies that we may have failed to identify around $7.4 \%$ of cases. This would be a problem only if differential identification occurred (identification of venous thromboembolism cases being more likely if they were treated with testosterone). However, this is unlikely to be the case and is unlikely to have altered the study findings.

Finally, despite the large number of more than 19000 venous thromboembolisms in the study population, only 69 cases were currently treated with testosterone, so subgroup analyses were based on even smaller numbers with decreased analytical power.

\section{Comparison with other studies}

The baseline (non-user) risk of venous thromboembolism and that after testosterone treatment, although increased transiently, are still relatively low in absolute terms. On an absolute population risk basis, the baseline and testosterone induced increases in risks of venous thromboembolism in this population of middle aged and older men are quantitatively comparable to the baseline (non-user) risks of venous thromboembolism in women and the increase among users of oral oestrogens in combined oral contraceptives or oestrogen replacement therapy for menopause. ${ }^{29-32}$ The early increase in risk in the first six months is also similar to that seen with oral oestrogen therapy. ${ }^{19}$ An increased risk of venous thromboembolism in patients with underlying familial or acquired thrombophilia if treated with testosterone has also been hypothesised and could explain the increased risk within six months after starting testosterone treatment. 33

\section{Meaning of study}

The fact that the risk peaks rapidly in the first three months and declines gradually thereafter indicates that failure to investigate the timing and duration of testosterone use could result in underestimation of the association between testosterone use and venous thromboembolism if overall risk estimates are based on a high proportion of patients carrying a small risk, a phenomenon known as depletion of susceptibles. ${ }^{26}$ This could explain the discrepancy between our findings and the recent case-control study. ${ }^{18}$ The mechanism for the transient increased risk due to testosterone treatment is uncertain but may involve effects on thrombosis via decreased fibrinolysis and an interaction with previously undiagnosed thrombophilia-hypofibrinolysis and/or with the higher underlying cardiovascular risk associated with erectile dysfunction. ${ }^{63435}$ The initial increased risk of venous clotting might provoke a secondary response with more fibrinolysis, which tends to dissolve clots and eventually neutralises the risk.
The risk of venous thromboembolism adjusted for underlying risk factors in association with testosterone use was increased early after the start of treatment for men without pathological hypogonadism, a group in which unproven empirical testosterone treatment has been increasingly used over the first decade of this century. ${ }^{136}$

These risks of venous thromboembolism associated with testosterone treatment are distinct from risks of other adverse arterial cardiovascular events associated with testosterone treatment in middle aged and older men as reported and reviewed elsewhere. ${ }^{112}$ Furthermore, venous thromboembolism should not be confused with pulmonary oil microembolisation, an immediate and transient effect of depot injections of testosterone esters in an oil vehicle. ${ }^{37}$

\section{Unanswered questions and future research}

Our study suggests a transient increase in the risk of venous thromboembolism that peaks during the first three to six months and declines gradually thereafter. Failure to investigate the timing of venous thromboembolisms in relation to the duration of testosterone use could result in masking of an existing transient association. Future research is needed to confirm this temporal increase in the risk of venous thromboembolism and to investigate the risk in first time testosterone users and confirm the absence of risk with long term use.

\section{Conclusions}

Starting testosterone treatment, whether first time or repeat use, is associated with an increased risk of venous thromboembolism that peaks rapidly in the first three months and declines gradually thereafter. This association is strengthened by a stronger association in the subgroup of patients without a known risk of venous thromboembolism. Overlooking the timing and duration of testosterone use in previous studies could have masked the association between testosterone use and cardiovascular events.

Contributors: CM, DJH, and SS were responsible for conception/ design of the study. AK, SR, and CM were responsible for data acquisition and analysis. All authors were involved in interpretation of data. CM and DJH were involved in drafting the manuscript, and all authors critically revised it for important intellectual content. CM is the guarantor.

\section{Funding: No external funding.}

Competing interests: All authors have completed the ICMJE uniform disclosure form at www.icmje.org/coi disclosure.pdf (available on request from the corresponding author) and declare: no support from any organisation for the submitted work; $\mathrm{CM}$ has received personal fees from Boehringer Ingelheim and grants from CSL Behring, Bayer Pharma AG, and Bristol-Myers Squibb; SS has received speaking fees from Novartis, Boehringer-Ingelheim, and AstraZeneca and research grants from Bayer Pharma AG, Boehringer-Ingelheim, Bristol-Myers Squibb, and Novartis; BF has received grants, personal fees, and non-financial support from Bayer Pharma AG, grants and non-financia support from Aspen, Boehringer Ingelheim, grants and personal fees from BMS/Pfizer, and personal fees from Servier, Astra-Zeneca, and Gilead. ATC has received grants and personal fees from Bayer HealthCare, Daiichi-Sankyo, Bristol-Myers Squibb, and Pfizer and personal fees from Boehringer Ingelheim, Johnson and Johnson, Ono Pharmaceuticals, Portola, Sanofi, X01, and Jannsen; DJH's institution received part funding from two companies (Besins, Lawley) in 2015 for investigator initiated clinical testosterone pharmacology research on which he is principal investigator, but he receives no personal funding; no other relationships or activities that could appear to have influenced the submitted work. 
Ethical approval: The study was approved by the Independent Scientific Advisory Committee for CPRD for Clinical Practice Research Datalink research (research No 14_154). No further ethics approval was required for the analysis of the data. The CPRD Group has obtained ethical approval from a multi-centre research ethics committee for all purely observational research using CPRD data; namely, studies that do not include patient involvement (the vast majority of CPRD studies). Data that could directly identify the patient were not collected in this study.

Data sharing: Clinical Practice Research Datalink data cannot be shared because of licensing restrictions.

Transparency: The lead author affirms that this manuscript is an honest, accurate, and transparent account of the study being reported; that no important aspects of the study have been omitted; and that any discrepancies from the study as planned (and, if relevant) have been explained.

This is an Open Access article distributed in accordance with the Creative Commons Attribution Non Commercial (CC BY-NC 3.0) license, which permits others to distribute, remix, adapt, build upon this work non-commercially, and license their derivative works on different terms, provided the original work is properly cited and the use is noncommercial. See: http://creativecommons.org/licenses/by-nc/3.0/.

1 Handelsman DJ. Global trends in testosterone prescribing, 2000-2011: expanding the spectrum of prescription drug misuse. Med J Aust 2013:199:548-51. doi:10.5694/mja13.10111.

2 Handelsman DJ. Androgen physiology, pharmacology and abuse. In: DeGroot LJ, Jameson JL, eds. Endocrinology. 7th ed. Elsevier Saunders, 2015: 2368-93.

3 Jasuja GK, Bhasin S, Reisman JI, Berlowitz DR, Rose AJ. Ascertainment of Testosterone Prescribing Practices in the VA. Med Care 2015;53:746-52. doi:10.1097/MLR.0000000000000398.

4 Handelsman D. Irrational Exuberance in Testosterone Prescribing When Will the Bubble Burst? Med Care 2015;53:743-5. doi:10.1097/ MLR.0000000000000416

5 Snyder PJ, Bhasin S, Cunningham GR, et al. Testosterone Trials Investigators. Effects of Testosterone Treatment in Older Men. N Engl J Med 2016;374:611-24. doi:10.1056/NEJMoa1506119.

6 Basaria S, Coviello AD, Travison TG, et al. Adverse events associated with testosterone administration. N Engl / Med 2010;363:109-22. doi:10.1056/NEJMoa1000485.

7 Albert SG, Morley JE. Testosterone therapy, association with age, initiation and mode of therapy with cardiovascular events: a systematic review. Clin Endocrinol (Oxf) 2016;85:436-43. doi:10.1111/cen.13084.

8 Araujo AB, Dixon JM, Suarez EA, Murad MH, Guey LT, Wittert GA. Clinical review: Endogenous testosterone and mortality in men: a systematic review and meta-analysis. / Clin Endocrinol Metab 2011;96:3007-19. doi:10.1210/jc.2011-1137.

9 Borst SE, Shuster JJ, Zou B, et al. Cardiovascular risks and elevation of serum DHT vary by route of testosterone administration: a systematic review and meta-analysis. BMC Med 2014;12:211. doi:10.1186/ s12916-014-0211-5.

10 Corona G, Maseroli E, Rastrelli G, et al. Cardiovascular risk associated with testosterone-boosting medications: a systematic review and meta-analysis. Expert Opin Drug Saf 2014;13:1327-51. doi:10.1517/14740338.2014.950653.

11 Ruige JB, Ouwens DM, Kaufman JM. Beneficial and adverse effects of testosterone on the cardiovascular system in men. J Clin Endocrinol Metab 2013;98:4300-10. doi:10.1210/jc.2013-1970.

12 Xu L, Freeman G, Cowling BJ, Schooling CM. Testosterone therapy and cardiovascular events among men: a systematic review and meta-analysis of placebo-controlled randomized trials. BMC Med 2013;11:108. doi:10.1186/1741-7015-11-108.

13 Onasanya O, Iyer G, Lucas E, Lin D, Singh S, Alexander GC. Association between exogenous testosterone and cardiovascular events: an overview of systematic reviews. Lancet Diabetes Endocrinol 2016:4:943-56. doi:10.1016/S2213-8587(16)30215-7.

14 US Food and Drug Administration. Testosterone products: FDA/CDER statement - risk of venous blood clots. 2014. http://www.fda.gov/Safety/ MedWatch/Safetylnformation/SafetyAlertsforHumanMedicalProducts/ ucm402054.htm.

15 Health Canada. Summary safety review - testosterone replacement products - cardiovascular risk 2014. http://www.hc-sc.gc.ca/ dhp-mps/medeff/reviews-examens/testosterone-eng.php.

$16 \mathrm{Xu} \mathrm{L}$, Schooling CM. Differential risks in men and women for first and recurrent venous thrombosis: the role of genes and environment: comment[comment]. / Thromb Haemost 2015;13:884-6. doi:10.1111/ jth.12862.
17 Sharma R, Oni OA, Chen G, et al. Association Between Testosterone Replacement Therapy and the Incidence of DVT and Pulmonary Embolism: A Retrospective Cohort Study of the Veterans Administration Database. Chest 2016;150:563-71. doi:10.1016/j. chest.2016.05.007.

18 Baillargeon J, Urban RJ, Morgentaler A, et al. Risk of Venous Thromboembolism in Men Receiving Testosterone Therapy. Mayo Clin Proc 2015;90:1038-45. doi:10.1016/j.mayocp.2015. 05.012.

19 Suissa S, Blais L, Spitzer WO, Cusson J, Lewis M, Heinemann L. First-time use of newer oral contraceptives and the risk of venous thromboembolism. Contraception 1997;56:141-6. doi:10.1016/ S0010-7824(97)00119-4.

20 Herrett E, Gallagher AM, Bhaskaran K, et al. Data Resource Profile: Clinical Practice Research Datalink (CPRD). Int J Epidemiol 2015:44:827-36. doi:10.1093/ije/dyv098.

21 Lawrenson R, Todd IC, Leydon GM, Williams T], Farmer RD. Validation of the diagnosis of venous thromboembolism in general practice database studies. Br J Clin Pharmacol 2000;49:591-6. doi:10.1046/i.1365-2125.2000.00199x.

22 Martinez C, Cohen AT, Bamber L, Rietbrock S. Epidemiology of first and recurrent venous thromboembolism: a population-based cohort study in patients without active cancer. Thromb Haemost 2014:112:255-63. doi:10.1160/TH13-09-0793.

23 Spencer FA, Emery C, Joffe SW, et al. Incidence rates, clinical profile, and outcomes of patients with venous thromboembolism. The Worcester VTE study. I Thromb Thrombolysis 2009;28:401-9. doi:10.1007/s11239-009-0378-3.

24 Samama MM, Cohen AT, Darmon JY, et al. Prophylaxis in Medical Patients with Enoxaparin Study Group. A comparison of enoxaparin with placebo for the prevention of venous thromboembolism in acutely ill medical patients. N Engl J Med 1999;341:793-800. doi:10.1056/NEJM199909093411103.

25 Suissa S. The Quasi-cohort approach in pharmacoepidemiology: upgrading the nested case-control. Epidemiology 2015;26:242-6. doi:10.1097/EDE.0000000000000221

26 Miettinen OS, Caro Il. Principles of nonexperimental assessment of excess risk, with special reference to adverse drug reactions. I Clin Epidemiol 1989;42:325-31. doi:10.1016/0895-4356(89)90037-1.

27 Schoenfeld MJ, Shortridge E, Cui Z, Muram D. Medication adherence and treatment patterns for hypogonadal patients treated with topical testosterone therapy: a retrospective medical claims analysis. J Sex Med 2013;10:1401-9. doi:10.1111/jsm.12114.

28 Rothman KJ. Lash TL Greenland S. Modern epidemiology. Revised 3rd ed. Lippincott-Raven, 2008

29 Dinger J, Bardenheuer K, Heinemann K. Cardiovascular and general safety of a 24-day regimen of drospirenone-containing combined ora contraceptives: final results from the International Active Surveillance Study of Women Taking Oral Contraceptives. Contraception 2014;89:253-63. doi:10.1016/i.contraception.2014.01.023.

30 Dinger IC, Heinemann LA, Kühl-Habich D. The safety of drospirenone-containing oral contraceptive: final results from the European Active Surveillance Study on oral contraceptives based on 142,475 women-years of observation. Contraception 2007:75:344-54 doi:10.1016/i.contraception.2006.12.019.

31 ESHRE Capri Workshop Group. Venous thromboembolism in women: a specific reproductive health risk. Hum Reprod Update 2013;19:471 82. doi:10.1093/humupd/dmt028.

32 Lidegaard $\varnothing$, Nielsen LH, Skovlund CW, Skjeldestad FE, Løkkegaard E. Risk of venous thromboembolism from use of oral contraceptives containing different progestogens and oestrogen doses: Danish cohort study, 2001-9. BMI 2011:343:d6423. doi:10.1136/bmi.d6423.

33 Glueck CJ, Prince M, Patel N, et al. Thrombophilia in 67 Patients With Thrombotic Events After Starting Testosterone Therapy. Clin Appl Thromb Hemost 2016;22:548-53 doi:10.1177/1076029615619486.

34 Winkler UH. Effects of androgens on haemostasis. Maturitas 1996;24:147-55. doi:10.1016/S0378-5122(96)82004-4

35 Glueck Cl, Goldenberg N, Budhani S, et al. Thrombotic events after starting exogenous testosterone in men with previously undiagnosed familial thrombophilia. Transl Res 2011;158:225-34. doi:10.1016/j. trsl.2011.06.003.

36 Perls T, Handelsman DJ. Disease mongering of age-associated declines in testosterone and growth hormone levels. J Am Geriatr Soc 2015;63:809-11. doi:10.1111/jgs.13391.

37 Middleton T, Turner L, Fennell C, et al. Complications of injectable testosterone undecanoate in routine clinical practice. EurJ Endocrinol 2015;172:511-7. doi:10.1530/EJE-14-0891.

\section{Supplementary table}

